\title{
Increased Dependence of Action Selection on Recent Motor History in Parkinson's Disease
}

\author{
Rick C. Helmich, ${ }^{1,3}$ Esther Aarts, ${ }^{1,2}$ Floris P. de Lange, ${ }^{1}$ Bastiaan R. Bloem, ${ }^{3}$ and Ivan Toni ${ }^{1,2}$ \\ Centres for ${ }^{1}$ Cognitive Neuroimaging and ${ }^{2}$ Cognition, Donders Institute for Brain, Cognition and Behaviour, Radboud University Nijmegen, and \\ ${ }^{3}$ Department of Neurology and Parkinson Centre Nijmegen (ParC), Radboud University Nijmegen Medical Centre, 6500 HB Nijmegen, The Netherlands
}

It is well known that the basal ganglia are involved in switching between movement sequences. Here we test the hypothesis that this contribution is an instance of a more general role of the basal ganglia in selecting actions that deviate from the context defined by the recent motor history, even when there is no sequential structure to learn or implement. We investigated the effect of striatal dopamine depletion [in Parkinson's disease (PD)] on the ability to switch between independent action plans. PD patients with markedly lateralized signs performed a hand laterality judgment task that involved action selection of their most and least affected hand. Trials where patients selected the same (repeat) or the alternative (switch) hand as in a previous trial were compared, and this was done separately for the most and least affected hand. Behaviorally, PD patients showed switch-costs that were specific to the most affected hand and that increased with disease severity. Functional magnetic resonance imaging (fMRI) showed that this behavioral effect was related to the state of the frontostriatal system: as disease severity increased, contributions of the basal ganglia to the selection process and their effective connectivity with the medial frontal cortex (MFC) decreased, whereas involvement of the MFC increased. We conclude that the basal ganglia are important for rapidly switching toward novel motor plans even when there is no sequential structure to learn or implement. The enhanced MFC activity may result either from reduced focusing abilities of the basal ganglia or from compensatory processes.

\section{Introduction}

The basal ganglia (BG) are important for set-shifting (Cools et al., 1984). For example, patients with Parkinson's disease (PD) are impaired when required to switch between movement sequences (Benecke et al., 1987; Harrington and Haaland, 1991; Hayes et al., 1998). These findings appear to fit with a more general role of the BG in updating internal representations (O'Reilly, 2006) and in switching toward novel behavior (Redgrave and Gurney, 2006). However, given the sequential structure of these previous motor tasks, and given the additional role of the BG in assembling performance units from sequence elements ("chunking") (Graybiel, 1998), impaired switching behavior in PD may result from both deficient chunking and updating. Using PD as a model for BG dysfunction, here we test the hypothesis that the BG have a general role in selecting actions that differ from the history of previously selected movements ("motor history"), even when there is no sequential structure to learn or implement (to prevent chunking).

Studies have shown that previously selected movements can influence current behavior over several seconds (Behrens et al.,

Received Feb. 11, 2009; revised March 20, 2009; accepted March 23, 2009.

This work was supported by the Alkemade-Keuls Foundation (to B.R.B.) and De Nederlandse Organisatie voor Wetenschappelijk Onderzoek (VIDI Grant No. 016.076.352 to B.R.B., VIDI Grant No. 452-03-339 to I.T.). We thank Roshan Cools and Pieter Medendorp for helpful comments on this manuscript, Paul Gaalman for his help with data collection, and Bram Daams for technical support.

Correspondence should be addressed to Dr. Rick Helmich, Centre for Cognitive Neuroimaging, Donders Institute for Brain, Cognition and Behaviour, Radboud University Nijmegen, P0 Box 9101, 6500 HB Nijmegen, The Netherlands. E-mail: Rick.Helmich@donders.ru.nl.

DOI:10.1523/JNEUROSCI.0704-09.2009

Copyright $\odot 2009$ Society for Neuroscience $\quad$ 0270-6474/09/296105-09\$15.00/0
2007; Rosenbaum et al., 2007), and some authors have conceptualized movement selection as either maintaining or changing a previous motor state (Polit and Bizzi, 1979; Rosenbaum et al., 2001). However, studies of actual motor performance cannot easily distinguish whether movement selection is influenced by the previous motor plan, or by the sensory consequences of having executed that plan. Here, we distinguish between these two possibilities by using motor imagery as a tool to evoke the internal generation of motor states, without explicit expression of those states (Jeannerod, 2006). Motor imagery has been extensively used to study action selection, an approach justified by empirical evidence showing that motor imagery is sensitive to motor control variables (Gentili et al., 2004) and uses neural operations involved in action planning (Cisek and Kalaska, 2004). We employed a motor imagery task in which subjects are asked to make laterality judgments (left or right) of hand pictures (Parsons, 1987), while measuring behavioral performance and cerebral activity [using functional magnetic resonance imaging (fMRI)]. Previous studies indicate that this task is sensitive to effectorspecific biomechanical constraints, both in healthy subjects (Shenton et al., 2004; de Lange et al., 2006) and in PD patients (Helmich et al., 2007). Crucially, instead of randomly selecting their left or right hand on each trial, in this task subjects consistently select the same hand as the stimulus on display (de Lange et al., 2008). This feature makes it possible to let PD patients internally select motor representations of either hand on a trial-bytrial basis, while experimentally biasing their choice by the laterality of the hand on display. Accordingly, we compared the behavioral and cerebral correlates of selecting (imagined) movements involving the same (repeat) or the alternative (switch) 
Table 1. Clinical characteristics (experiments 1 and 2)

\begin{tabular}{|c|c|c|c|c|c|c|c|c|c|c|}
\hline \multirow[b]{2}{*}{ Patient } & \multirow[b]{2}{*}{ Experiment } & \multirow[b]{2}{*}{ Gender } & \multirow[b]{2}{*}{ Age } & \multicolumn{6}{|c|}{ UPDRS part III (motor section) } & \multirow[b]{2}{*}{$H \& Y$} \\
\hline & & & & Right hand & Left hand & Right foot & Left foot & Other & Total & \\
\hline 1 & 2 & 2 & 55 & 11 & 7 & 5 & 3 & 6 & 32 & 2 \\
\hline 2 & 1 & 1 & 39 & 13 & 3 & 4 & 0 & 6 & 26 & 2 \\
\hline 3 & 1 & 1 & 48 & 15 & 3 & 3 & 1 & 16 & 38 & 2.5 \\
\hline 4 & 1 & 2 & 51 & 8 & 5 & 4 & 2 & 8 & 27 & 2 \\
\hline 5 & 1 & 1 & 48 & 10 & 3 & 5 & 0 & 14 & 32 & 3 \\
\hline 6 & 1 & 1 & 53 & 8 & 1 & 2 & 1 & 7 & 19 & 2 \\
\hline 7 & 1 & 1 & 50 & 8 & 1 & 3 & 1 & 7 & 20 & 1.5 \\
\hline 8 & $1-3$ & 2 & 59 & 11 & 5 & 4 & 1 & 7 & 28 & 2 \\
\hline 9 & $1-3$ & 1 & 68 & 12 & 5 & 3 & 3 & 7 & 30 & 2 \\
\hline 10 & $1-3$ & 1 & 65 & 5 & 2 & 3 & 1 & 3 & 14 & 2 \\
\hline 11 & $1-3$ & 1 & 56 & 10 & 5 & 4 & 2 & 2 & 23 & 2 \\
\hline 12 & $1-3$ & 1 & 34 & 10 & 0 & 4 & 0 & 8 & 22 & 1.5 \\
\hline 13 & $1-3$ & 1 & 50 & 10 & 5 & 4 & 2 & 13 & 34 & 3 \\
\hline 14 & $1-3$ & 1 & 53 & 11 & 3 & 4 & 2 & 7 & 27 & 2 \\
\hline 15 & $1-3$ & 2 & 67 & 8 & 2 & 6 & 4 & 5 & 25 & 2 \\
\hline 16 & $1-3$ & 1 & 65 & 4 & 0 & 2 & 0 & 6 & 12 & 1.5 \\
\hline 17 & 1,3 & 2 & 43 & 13 & 8 & 3 & 1 & 6 & 31 & 2 \\
\hline 18 & 1,3 & 2 & 56 & 10 & 3 & 1 & 1 & 4 & 19 & 2 \\
\hline Mean & & 12 men & 53.3 & 9.8 & 3.4 & 3.6 & 1.4 & 7.3 & 25.5 & 2.1 \\
\hline SD & & & 9.4 & 2.7 & 2.3 & 1.2 & 1.1 & 3.6 & 7.0 & 0.4 \\
\hline
\end{tabular}

Eighteen patients with idiopathic Parkinson's disease were tested in a practically-defined off-state [more than $12 \mathrm{~h}$ after having taken their last medication (Langston et al., 1992)]. All patients were consistent right-handers. Patients had markedly asymmetric symptoms lateralized to the right side of their body, and more symptoms in their hands than in their feet. Different patients participated in different experiments (see second column). Patients $8-18$ were retested approximately 1 year later when participating in experiment 3 (for clinical scores, see supplemental Table 1, available at www.jneurosci.org as supplemental material). Highest possible UPDRS-III score is 108 points. The highest possible UPDRS score for each side is 36 points: 24 points for the hand and 12 points for the foot. H\& Y, Hoehn and Yahr rating scale (highest stage is 5). Gender: 1, man; 2, woman.

hand with respect to the previous trial, and this was done separately for the most and least affected hand. Importantly, withinpatient comparisons allowed us to control for perceptual discrimination processes and for nonlateralized PD-related pathologies (Eidelberg et al., 1995; Kaasinen et al., 2000).

\section{Materials and Methods \\ Subjects}

Parkinson patients. Eighteen right-handed idiopathic PD patients (12 men, $53 \pm 3$ years, mean $\pm \mathrm{SD}$ ) participated after having given written informed consent according to institutional guidelines of the local ethics committee. Patients were included when they had idiopathic PD, diagnosed according to the UK Brain Bank criteria by an experienced movement disorders specialist (B. R. Bloem), all with clearly right-lateralized symptoms. Exclusion criteria were: moderate-severe tremor, cognitive dysfunction (Mini Mental State Examination $<24$ or frontal executive problems), other neurological diseases, and general exclusion criteria for MRI scanning. Frontal executive problems were assessed clinically using the Frontal Assessment Battery (FAB) (Dubois et al., 2000) before patients were included in this study. Only patients with no or very mild executive impairments were included (FAB $>$ or equal to 16 ; maximum score is 18 points). Five patients had never used any anti-Parkinson medication; the others used levodopa or dopamine-agonists. The experiments were performed in the morning, at least $12 \mathrm{~h}$ after the last dose of dopaminergic medication (Langston et al., 1992). Disease severity was assessed using the Hoehn and Yahr stages and the motor section of the Unified Parkinson's Disease Rating Scale (UPDRS-III; Table 1). All patients showed markedly right lateralized signs (UPDRS-right: $13.4 \pm 3.1$; UPDRS-left: $4.8 \pm 2.9$; average $\left.\pm \mathrm{SD} ; t_{(17)}=12.1 ; p<0.001\right)$. To directly compare the UPDRS scores for the hands and the feet, we had to correct for the fact that there are more hand- than foot-specific UPDRS items. Thus, the UPDRS-foot score was calculated as the sum of items 20 (resting tremor), 22 (rigidity), and 26 (bradykinesia). The UPDRS-hands score was calculated as the sum of items 20 (resting tremor), 22 (rigidity), and the average of items 23-25 (all referring to bradykinesia). Comparison of normalized UPDRS scores across "effector" (hand, foot) and "laterality" (left, right) revealed greater disease severity for the hands than for the feet (UPDRS-hands: 6.4 \pm 0.5 ; UPDRS-feet: $4.9 \pm 0.5$; main effect of effector: $\left.F_{(1,17)}=7.2, p=0.016\right)$, greater disease severity for the right than for the left side (UPDRS-right: $8.4 \pm 0.4$ UPDRS-left: $3.0 \pm$
0.4; main effect of laterality: $\left.F_{(1,17)}=174.2, p<0.001\right)$, and a greater asymmetry for the hands than for the feet (effector $\times$ laterality interaction: $\left.F_{(1,17)}=5.8, p=0.028\right)$.

Healthy subjects. Ten healthy, right-handed control subjects participated in experiment 2 . The distributions of age (PD: $53 \pm 3$ years, controls: $57 \pm 6$ years; $t_{(26)}=-1.1, p=0.38$, independent-samples $t$ test) and gender (PD: 12 men and 6 women; controls: 6 men and 4 women; $t_{(26)}=-0.34, p=0.74$, independent-samples $t$ test) were similar across the two groups.

\section{Experimental tasks}

Experiment 1. Patients were lying supine in an MR scanner, facing the bore of the magnet, unable to see their hands, in front of a mirror facing a projection screen (at the back of the MR scanner), with their left and right big toes strapped onto separate MR compatible response buttons. They were presented with line drawings of left and right hands (Fig. 1), and they were asked to report as quickly as possible whether they saw a picture of a left or right hand by flexing their left or right big toe, respectively. Reaction time was defined as the time between onset of the visual stimulus and key press. Each imagery trial started with a fixation cross, displayed for a variable interval, followed by the presentation of a hand drawing. When a response was provided, the stimulus was replaced by the fixation cross until presentation of the next hand drawing. The intertrial interval between the offset of one trial (i.e., the response) and the onset of the next (i.e., the presentation of the stimulus) varied randomly between 1.5 and $2.5 \mathrm{~s}$, creating a random jitter between successive trials. In addition, the trials were presented in a pseudo-randomized order, which ensured minimal correlations between different event types (i.e., the different conditions). This led to a maximum correlation of $19.5 \%$ between the task regressors. Furthermore, within the framework of the general linear model all regressors are implicitly orthogonalized to each other, resulting in each regressor only explaining the variance uniquely attributed to it (Friston, 2007). There was a reaction time cutoff at $5.0 \mathrm{~s}$. The stimuli subtended a visual angle of $\sim 10^{\circ}$.

The left- and right-hand stimuli were identical mirror images, they could be shown rotated from an upright $\left(0^{\circ}\right)$ position from $-135^{\circ}$ to $+135^{\circ}$ in eight steps of $45^{\circ}$, and they could be shown as back or palm views, according to a pseudo-randomized trial order (Fig. 1). Hand rotation and hand orientation were not of primary interest for this study, but their inclusion in the experimental design was important to ensure 


\section{Factor HAND}

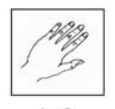

left

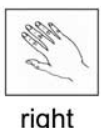

right
Factor HAND-ORDER

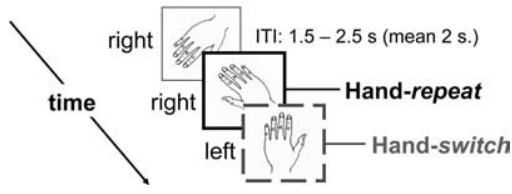

Figure 1. Task setup (experiments 1 and 2). For each trial, subjects had to judge whether a visually presented drawing showed a left or right hand. The top panel illustrates two representative stimuli, one for each hand laterality. Crucially, for each trial we considered whether the previous stimulus had the same or a different laterality than the currently displayed hand drawing. Accordingly, we analyzed the effect of factors hand (2 levels: left or right) and handorder (2 levels: repeat or switch) on reaction times and cerebral activity. The intertrial interval (ITI) between the offset of one trial (i.e., the response) and the onset of the next (i.e., the presentation of the stimulus) randomly varied between 1.5 and $2.5 \mathrm{~s}$, creating a pseudorandom jitter between successive trials.

that subjects used motor imagery to solve the hand laterality judgment task, rather than alternative processes like spatial mappings between position of a hand feature and hand laterality. There were 480 stimuli, presented in 30 imagery blocks of 16 trials, with a total measurement time of $\sim 40 \mathrm{~min}$. Each imagery block was followed by a $10 \mathrm{~s}$ baseline period (fixation cross-display). The main effects of stimulus rotation and hand laterality on reaction times and cerebral activity have been described in a previous study on the same data set (Helmich et al., 2007). Here, the focus is on trial order effects in the laterality of imagined hand movements: the effect of laterality of the hand imagined moving at trial $n-1$ on imagery performance at trial $n$ (Fig. 1). This effect was measured by sorting the hand laterality (left or right) at each trial as a function of the hand laterality at the previous trial. When trial $n-1$ had the same hand laterality as trial $n$ (e.g., images of left hands were presented at trial $n-1$ and at trial $n$ ), then trial $n$ was defined as a repeat trial. Conversely, when trial $n-1$ had a different laterality as trial $n$ [e.g., an image of a left hand (at trial $n-1$ ) was followed by an image of a right hand (at trial $n$ )], then trial $n$ was defined as a switch trial. The first trial of each of the 30 blocks, incorrect trials, and trials following incorrect trials were excluded from the analysis of the trial order effects.

Experiment 2. To confirm that the lateralized hand-switch effect found in experiment 1 was specific to $\mathrm{PD}$, we performed a control experiment (involving the same motor imagery task; see supplemental Methods, available at www.jneurosci.org as supplemental material) comparing performance of repeat and switch trials between a group of $10 \mathrm{PD}$ patients (Table 1) and 10 matched, healthy controls.

Experiment 3. To confirm that the lateralized hand-switch effect found in experiment 1 was specific to internally generated motor plans, we performed a control experiment (involving externally cued hand movements; see supplemental Methods, available at www.jneurosci.org as supplemental material) in $11 \mathrm{PD}$ patients (see Table 1) comparing performance of repeat and switch trials between their most and least affected hand. In this experiment, patients were asked to make a movement with either their left or their right hand to a target positioned on either the left or the right side of the table. Movements were always congruent (i.e., left hand to left target and right hand to right target). Movements were triggered by either a light or a sound, and the light-emitting diode and loudspeaker were positioned directly onto the target buttons. Thus, in experiment 3 there was a direct spatial mapping between the stimulus and the required movement (high stimulus-response compatibility), which effectively minimized the load on internal selection processes. Conversely, the low stimulus-response compatibility in experiments 1 and 2 ensured that patients had to internally select the appropriate effector (left or right hand) for subsequent motor imagery. Note that in all three experiments the timing (or onset) of the (imagined) actions was always triggered by the presentation of a stimulus. This procedure ensured that differences between the two sets of experiments were specific to the "what-component" of internal selection (Mueller et al., 2007), while minimizing the load on the "when-component" of internal selection (Jahanshahi et al., 1995; Jahanshahi et al., 1998).

\section{Image acquisition and processing}

Functional images were acquired on a Siemens SONATA 1.5 T MRI system (Siemens) equipped with echo planar imaging (EPI) capabilities, using the standard head coil for radio frequency transmission and signal reception. Blood oxygenation level dependent (BOLD) sensitive functional images were acquired using a single shot gradient EPI sequence [echo time (TE)/repetition time (TR) $=40 / 2560 \mathrm{~ms} ; 32$ axial slices, voxel size $=3.5 \times 3.5 \times 3.5 \mathrm{~mm}$; field of view $(F O V)=224 \mathrm{~mm}]$. High resolution anatomical images were acquired using an MP RAGE sequence $(\mathrm{TE} / \mathrm{TR}=3.39 / 2250 \mathrm{~ms}$; voxel size $=1.0 \times 1.0 \times 1.0 \mathrm{~mm}, 176$ sagittal slices; FOV $=256 \mathrm{~mm})$. Functional data were preprocessed and analyzed with SPM2 (Statistical Parametric Mapping, www.fil.ion. ucl.ac.uk/spm). The same preprocessing procedures described by Helmich et al. (2007) were used, as described in the supplemental Methods, available at www.jneurosci.org as supplemental material.

\section{Statistical model}

Our statistical model on the first (subject specific) level considered the laterality of the hand drawing on display (factor "hand," two levels: most affected or least affected) and the order of left and right-hand stimuli (factor "hand-order," two levels: repeat or switch). We also included a factor of no interest describing stimulus-driven switches between the back and the palm of the hand (factor "orientation-order," two levels: orientation-repeat or orientation-switch). We collapsed over this factor in subsequent random effect analyses. Only correct responses were included in these regressors, with trials modeled as square-wave functions time-locked to stimulus onset, and durations corresponding to the mean reaction time across all imagery trials of the subject. Finally, our model included separate regressors of no interest, modeling the trials at the beginning of each block, incorrect and missed responses, residual head movement-related effects, global signal changes (as indexed by segmented white matter and cerebral spinal fluid), and low frequency signal drifts over time (period $>60 \mathrm{~s}$ ). Residual head movement effects were modeled by including a Volterra expansion of the six rigid body motion parameters as nuisance covariates in the design matrix of the GLM (Lund et al., 2005). The Volterra expansion consisted of linear, quadratic, and cubic effects of the six movement parameters belonging to each volume and also the first and second derivative of each of those regressors (to control for spin history effects), giving rise to a total of 54 movement regressors. Parameter estimates for all regressors were obtained by maximum-likelihood estimation, modeling temporal autocorrelation as a first-order autoregressive model $[\mathrm{AR}(1)]$ process.

On the second (random effects) level we performed a within-patient ANOVA with factors hand (most affected, least affected) and hand-order (repeat, switch), together with an additional regressor modeling "incorrect" trials. First, we searched for brain regions in which activity changed as a function of hand-order (switch $>$ repeat or repeat $>$ switch), separately for each hand. Then we investigated whether the activity in these regions changed as a function of hand by performing an inclusive masking with the interaction term (i.e., hand $\times$ hand-order). This revealed brain regions in which switch-related activity was higher for the most affected hand than for the least affected hand. Second, we searched for brain regions showing error-related activity (incorrect $>$ all correct regressors) and we tested for overlap between switch- and error-related brain activity by performing a conjunction analysis (Nichols et al., 2005) between the previous two contrasts.

We report the results of a random effects analysis, correcting for multiple comparisons at the cluster level $(p<0.05)$, based on a voxel level intensity threshold of $p<0.001$ uncorrected (Friston et al., 1996).

\section{Post hoc analyses}

The results of the contrasts described above indicated a double dissociation between "region" [cingulate motor area (CMA) vs anterior cingulate cortex (ACC)] and "task" (switching vs error processing). To formally 
test this effect, we extracted the $\beta$ values of the multiple regression from the local maxima within the CMA ([4-244]) (see Fig. 3A-C) and the ACC ([2 2636$])$ (Fig. 3D-F). Then, we computed activity related to switching (difference between the $\beta$ values for switch and repeat conditions) and to error processing (difference between the $\beta$ values for incorrect and correct conditions) in each region, and we performed a repeated measures ANOVA with factors region (CMA vs ACC) and task (switching vs error processing) on these difference scores. Four post hoc $t$ tests were performed to test for switch- and error-related activity in the CMA and the ACC.

\section{Brain-disease relationship}

To test for an effect of disease severity on switch-related brain activity, we performed a simple regression analysis correlating patient-specific switch-related activity (for the most affected hand; contrast images obtained at the first level) voxel-by-voxel with lateralized disease severity (for the most affected side, in UPDRS points). We focused our analyses on the medial frontal cortex [volume of interest (VOI) of $4.1 \mathrm{ml}$, showing switch-related activity for the most affected hand in the random effects analysis described above], as well as the bilateral BG [globus pallidus and putamen; four VOIs taken from the automated anatomical labeling (AAL) atlas (Tzourio-Mazoyer et al., 2002)]. In these regions, we corrected for multiple comparisons using the same statistical threshold described above.

Similarly, to test for an effect of disease severity on switch-related effective connectivity between BG and the CMA, we performed a simple regression analysis correlating patient-specific effective connectivity maps (describing larger connectivity for switch than repeat trials, specifically for the most affected hand; psycho-physiological interaction analysis, see supplemental Methods, available at www.jneurosci.org as supplemental material) with lateralized disease severity (for the most affected side, in UPDRS points).

This resulted in four significant brain-disease correlations in three regions: the CMA, the left GP, and the left putamen. To test whether these correlations were specifically driven by disease severity of the most affected hand (given that the task involved hand and not foot imagery), for each of these three regions we additionally calculated the partial correlation between disease severity of the most affected hand (sum of UPDRS items $20-25$ ) and switch-related cerebral activity/connectivity ( $\beta$ values taken from each region's local maximum), while controlling for disease severity of the most affected foot (sum of UPDRS items 20,22, and 26). Similarly, for each of these three regions we performed the same procedure to assess the unique correlation between disease severity of the most affected foot and switch-related cerebral activity/connectivity, while controlling for disease severity of the most affected hand.

\section{Results}

\section{Experiment 1}

In this experiment, we compared performance of switch and repeat trials for the most and least affected hand in a within-patient design. We predicted specific impairments for switch trials involving the most affected hand. First, we searched for brain regions showing larger switch-related activity for the most affected hand than for the least affected hand. Second, we assessed the functional specificity of the switch-related responses by distinguishing them from performance monitoring effects (as indexed by error-related processing). Third, we investigated how switchrelated cerebral activity and frontostriatal effective connectivity changed as a function of disease severity.

\section{Behavioral performance}

Patients performed the task accurately, with a mean error rate $( \pm$ SEM) of $7.6 \pm 1.6 \%$.

They had difficulties switching from the least affected hand to the most affected hand, but not vice versa (hand $\times$ hand-order interaction: $F_{(1,16.4)}=6.22 ; p=0.024$; main effect of hand-order for the most affected hand: $F_{(1,16.4)}=5.43 ; p=0.033$; no main

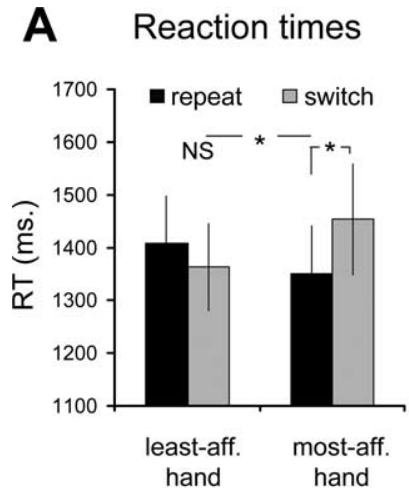

\section{B Most-affected hand}

Figure 2. Behavioral performance (experiment 1). The bars show the time that patients used to solve the hand-laterality judgment task (RT, in milliseconds; mean \pm SEM). RTs for repeat (black bars) and switch trials (gray bars) are shown separately according to hand laterality (least affected, most affected). $\boldsymbol{A}$ shows RTs from 17 PD patients during fMRI scanning. $\boldsymbol{B}$ shows how the size of the behavioral switch cost for the most affected hand (on the $y$-axis, calculated as the difference between the averaged log-transformed RT for switch trials and repeat trials) increases linearly with disease severity (for the most affected side, on the $x$-axis). Each dot represents one patient. ${ }^{*}$ and NS indicate statistically significant and nonsignificant effects, respectively.

effect of hand-order for the least affected hand: $\mathrm{F}_{(1,16.6)}=0.75$; $p=0.40$; Figure $2 A$ ). In addition, behavioral switch costs were linearly related to disease severity: the more impaired the affected side, the larger the behavioral switch cost $(r=0.50 ; p=0.040)$ (Fig. $2 B$ ).

\section{Switch-related brain activity}

We found larger switch-related activity for the most affected hand than for the least affected hand (hand $x$ hand-order interaction) in one cluster located within the medial frontal cortex (MFC; $p<0.001$ corrected for multiple comparisons) (Fig. $3 A, B)$. This cluster covered the CMA (local maximum at $[4-2$ $44]$ ) and the pre-SMA (local maximum at [ -4852$]$ ) (Picard and Strick, 1996). We ruled out that this activity pattern was driven by the longer reaction times (RTs) during switch trials, because it was equally present when correcting for trial-by-trial RT differences (local maximum at $[4-642], p=0.006$ corrected for multiple comparisons; see supplemental Fig. 2, available at www. jneurosci.org as supplemental material). There were no brain regions that showed significantly larger switch-related cerebral activity for the least affected hand and there were no brain regions showing larger activity for repeat than switch trials.

\section{Error-related brain activity}

The switch-related activity described above falls in the MFC, anterior parts of which have been associated with cognitive control processes (e.g., performance adjustments following errors (Ridderinkhof et al., 2004)). To test whether the switch-related activity described above simply reflected increased cognitive control, we compared the size and the spatial distribution of error- and switch-related brain activity within the same data set. Given that patients did not receive feedback about their performance, the error-related activity assessed here relates to internal performance monitoring rather than feedback processing (Mars et al., 2005).

In line with previous reports, we found stronger responses during erroneous trials than during correct trials in the anterior MFC $(p<$ 0.001 corrected; other regions with error-related responses are listed in supplemental Table 1, available at www.jneurosci.org as supple- 

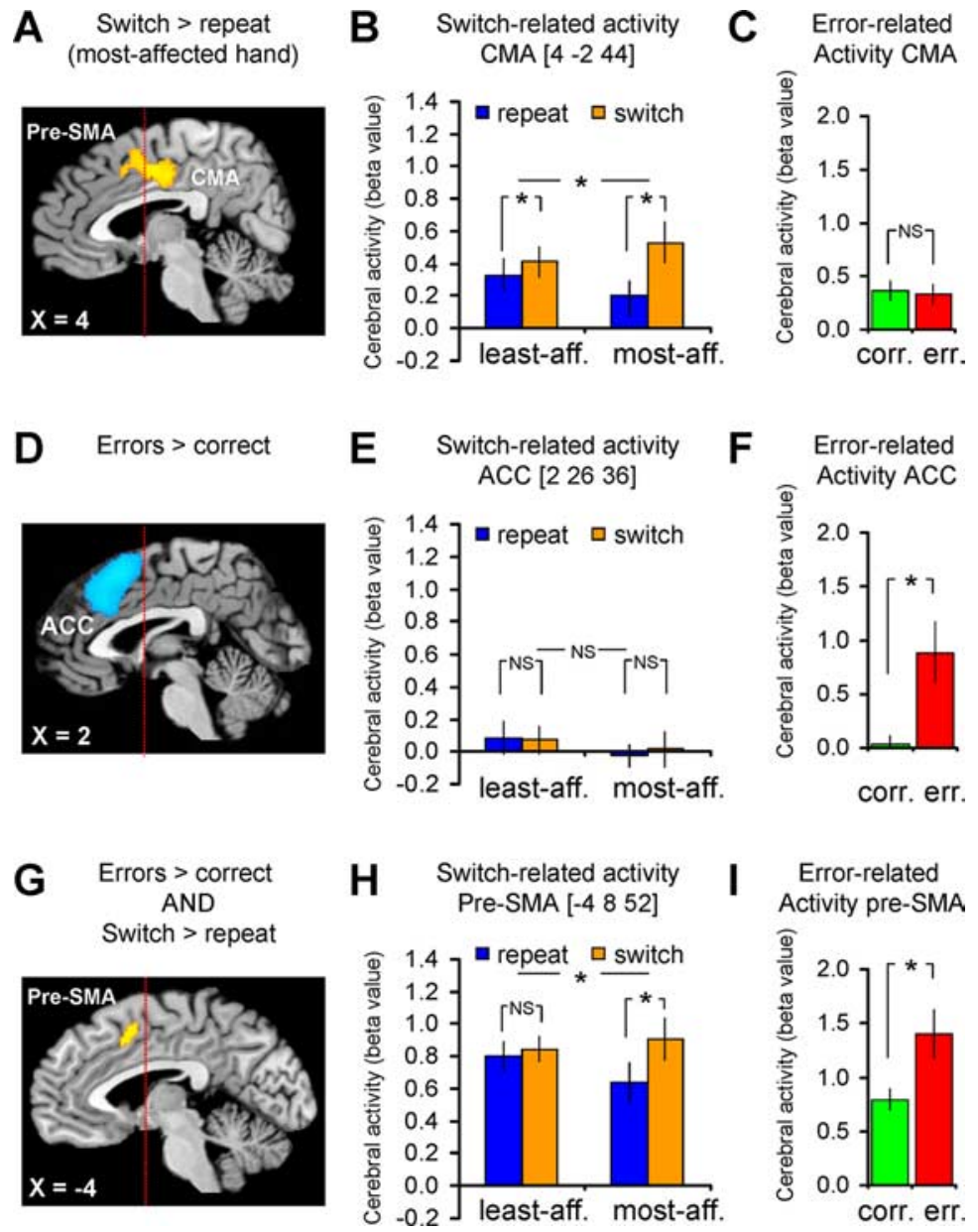

Figure 3. Switch- and error-related brain activity. The left column shows the anatomical distribution of switch- and error-related activity, the middle column shows the effects size of switch-related cerebral responses and the right column shows the effects size of error-related responses. $A-C$, Brain regions in which cerebral activity increased during hand-switch trials (compared with hand-repeat trials), specifically for the most affected hand (compared with the least affected hand). $\boldsymbol{D}-\boldsymbol{F}$, Brain regions in which cerebral activity increased during error trials (compared with correct trials). $G-I$, Brain regions that were sensitive to both switch- and error-related effects [conjunction analysis of the overlap between switch-related effects $(\boldsymbol{A}-\boldsymbol{C})$ and error-related effects $(\boldsymbol{D}-\boldsymbol{F})$ (Nichols et al., 2005)]. These results show a double dissociation in the response profiles of the CMA and the ACC to switching and error processing: the CMA [the posterior part of the switch-related cluster within the $\operatorname{MFC}(\boldsymbol{A})$ ] responded only during switch trials $(\boldsymbol{B})$, but not during error trials $(\boldsymbol{C}$ ). In contrast, the ACC [the anterior part of the error-related cluster within the $\operatorname{MFC}(\boldsymbol{D})$ ] responded only during error trials $(\boldsymbol{F})$, but not switch trials $(\boldsymbol{E})$. The pre-SMA $(\boldsymbol{G})$ showed both switch- $(\boldsymbol{H})$ and error-related activity $(\boldsymbol{I})$. The statistical parametric maps $(\boldsymbol{A}, \boldsymbol{D}$, G) represent the results of a random effects analysis, shown at an uncorrected threshold of $p<0.001$ (for graphical purposes), and superimposed on sagittal sections of a representative brain of the Montreal Neurological Institute series. The histograms $(\boldsymbol{B}, \boldsymbol{E}, \boldsymbol{H}$ and $\boldsymbol{C}, \boldsymbol{F}, \boldsymbol{I})$ show the mean ( \pm SEM) parameter estimates from this random effects analysis.

mental material). This MFC cluster spanned both pre-SMA (local maximum at [ $\left.\begin{array}{lll}-2 & 18 & 50\end{array}\right]$ and ACC (local maximum at [2 26 36]) (Fig. 3D), but it did not extend into the CMA (Fig. 3C).

These results show a double dissociation in the response profiles of the CMA and the ACC to switching and error processing [region $(\mathrm{CMA}$ vs ACC) $\times$ task (switching vs error processing) interaction: $\left.F_{(1,16)}=15.27 ; p=0.001\right]$. That is, the CMA (the posterior part of the switch-related cluster within the MFC) responded only during switch trials (CMA-switching: $t_{(16)}=4.76$, $p<0.001$ ), but not during error trials (CMA-error processing: $t_{(16)}=-0.30, p=0.77$ ). In contrast, the ACC (the anterior part of the error-related cluster within the MFC) responded only during error trials (ACC-error processing: $t_{(16)}=3.67, p=0.002$ ), but not switch trials (ACC-switching: $t_{(16)}=0.49, p=0.63$ ) (Fig. 3).

The pre-SMA was unique in showing both switch- and errorrelated activity, as assessed by means of a conjunction analysis
(Nichols et al., 2005) ( $p=0.037$ corrected; local maximum at $\left.\left[\begin{array}{lll}-4 & 8 & 52\end{array}\right]\right)$ (Fig. $3 G$ ). That is, the pre-SMA responded to both switch trials (preSMA-switching: $\left.t_{(16)}=3.8, p=0.002\right)$ and error trials (pre-SMA-error processing: $\left.t_{(16)}=3.7, p=0.002\right)$.

Relationship between cerebral effects and disease severity

The positive correlation between lateralized switch cost and lateralized disease severity (Fig. 2B) had a cerebral counterpart in the same portion of the MFC showing specific switch-related cerebral responses (CMA, $r=0.75, p=$ 0.01 corrected, local maximum at $[4$ $-246]$ ) (Fig. 4A,B). There was no correlation between switch-related brain activity and disease severity in the preSMA $(r=0.11, p=0.65$, switch-related responses taken from the local maximum at $\left[\begin{array}{lll}-4 & 8 & 52\end{array}\right]$ ) (Fig. 3G-I). This indicates that the brain-disease relationship was spatially specific, occurring only in the CMA, but not in the pre-SMA [ $r$ (pre-SMA) vs $r$ (CMA): $t_{(14)}$ $=4.22 ; p<0.001, t$ test for comparison of two dependent correlations from the same sample (Chen and Popovich, 2002)].

In contrast, switch-related activity in the most affected GP was negatively correlated with lateralized disease severity (left rostromedial GP, $r=-0.74$, $p=0.04$ corrected, local maximum at $[-18-2-2]$ ) (Fig. 4C,D). Together, the clinically least impaired patients showed the highest switch-related activity in the most affected GP, and little if any switch-related activity in the CMA. Conversely, the most affected patients showed no (or negative) switch-related activity in the GP, and robust switch-related activity in the CMA. These findings indicate that the contribution of these regions to switching was inversely related (Fig. 4). The effects were spatially specific: there were no significant correlations in the least affected BG. The brain-disease correlations in the CMA and in the left GP were specific to disease severity in the most affected hand and not related to disease severity in the most affected foot (see Table 2 for post hoc partial correlations).

Last, given the dense connections between the CMA and the BG [particularly the anterior putamen (McFarland and Haber, 2000; Takada et al., 2001)] and given the detrimental effect of dopamine depletion on corticostriatal connectivity (Lalo et al., 2008), we directly tested whether effective connectivity between these structures would change as a function of disease severity. Switch-related effective connectivity between the CMA and the most affected BG significantly decreased as a function of lateralized disease severity (left GP, $r=-0.71, p=0.039$ corrected, local maximum at $[-2042]$; left putamen, $r=-0.80, p=0.007$ 
corrected, local maximum at [ -224 2]) (Fig. $4 E, F)$. These effects were spatially specific: they were not present for the least affected BG and they were localized to the anterior putamen, which is anatomically connected to the CMA (Takada et al., 2001) and specifically involved in internally guided action selection (Jueptner et al., 1997). Again, these correlations were specific to disease severity in the most affected hand and not related to disease severity in the most affected foot (Table 2).

\section{Experiment 2}

To test whether the switch effects described above were specific to PD, we directly compared behavioral performance of $10 \mathrm{PD}$ patients with that of 10 matched healthy controls during a task similar to experiment 1 (see supplemental material, available at www. jneurosci.org). There were significant switch-costs only in the PD group (group $\times$ hand-order interaction: $F_{(1,18)}=14.60 ; p=0.001$; main effect of hand-order for PD patients: $F_{(1,9)}=$ 20.76; $p=0.001$; no main effect of hand-order for controls: $F_{(1,9)}=1.44$; $p=0.26)$. This confirms that the switch effects described in experiment 1 were specific to $\mathrm{PD}$.

\section{Experiment 3}

To test whether the switch effects described above were specific to internally generated movements (compared with spatially cued movements), we performed a behavioral control experiment in which patients executed spatially cued hand movements, comparing switch and repeat trials for the most and least affected hand (see supplemental material, available at www. jneurosci.org). There was no significant effect of hand-order, and no hand $\times$ hand-order interaction $\left(F_{(1,10)}=0.19 ; p=0.67\right)$. This indicates that the switch effects described in experiments 1 and 2 are specific to internally generated movements.

\section{Discussion}

We investigated the effect of striatal dopamine depletion on the ability to switch between independent action plans, as evoked by a motor imagery task that lacked any predefined structure across trials. There are two main findings. First, PD patients were specifically impaired in action selection of their most affected hand, but only when this involved a switch with respect to the hand selected in the previous trial. Cerebrally, this effect was accompanied by decreased activity in the most affected globus pallidus. This finding supports our hypothesis that the BG have a specific role in action selection when a switch from recent motor history is required. Second, we show enhanced switch-related activity in the medial frontal cortex that increased with advancing disease.
B Cingulate Motor Area [4 -2 46]

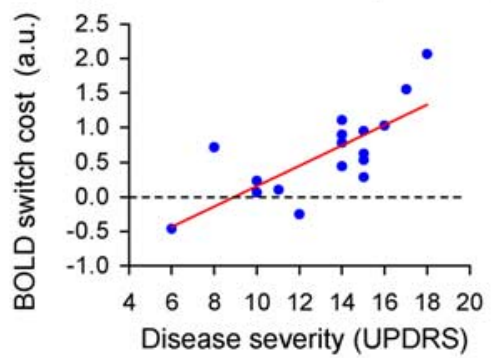

D Left Globus Pallidus [18 -2 -2]

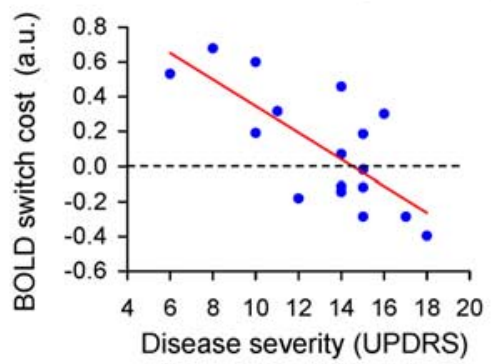

F Left Putamen [-22 4 2] $\leftrightarrow$ CMA

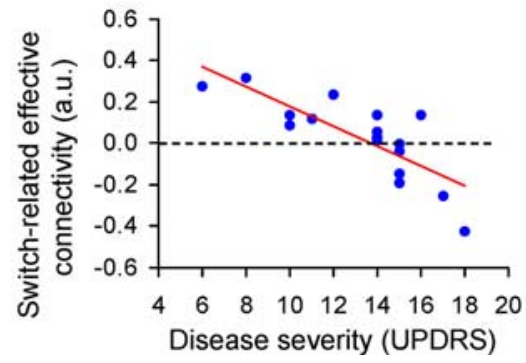

Figure 4. Correlation between cerebral effects and disease severity. $\boldsymbol{A}, \boldsymbol{C}, \boldsymbol{E}$ show the anatomical distribution of switch-related responses that were modulated by clinical disease severity. $\boldsymbol{B}, \boldsymbol{D}, \boldsymbol{F}$ show the relationship between switch-related activity and disease severity. Switch-related responses and disease severity are shown for the most affected side. $\boldsymbol{A}, \boldsymbol{B}$, Brain regions in which switch-

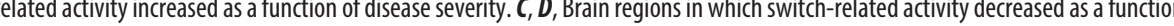
of disease severity. $\boldsymbol{E}, \boldsymbol{F}$ show that switch-related interregional coupling between the CMA ( $\boldsymbol{E}$, in orange) and the left putamen/GP ( $\boldsymbol{E}$ decreased as a function of disease severity. In $\boldsymbol{B}$ and $\boldsymbol{D}$, each dot represents switch-related activity for one patient. In $\boldsymbol{E}$, each do

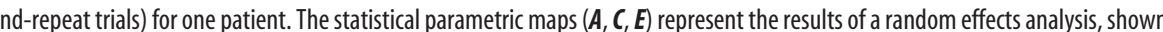
at an uncorrected threshold of $p<0.01$ (for graphical purposes), and superimposed on sagittal and coronal sections of a representative brain of the Montreal Neurological Institute series. The left side of the figure shows the left side of the brain.

This pattern of cortical responses may result either from functional compensation, or from reduced focusing within the frontostriatal circuit.

\section{The effect of striatal dysfunction on action switching}

The hand-switch effect we report was functionally specific in three different aspects. First, the effect was present in PD patients, not in healthy controls, and it was related to disease severity. Second, the hand-switch effect was lateralized toward the most affected hand, but absent (or considerably reduced) for the least affected hand. This asymmetry rules out several potential confounds that were matched across hands, such as perceptual changes in stimulus features during switch trials, nonlateralized cognitive impairments [attention, working memory (Mattay et al., 2002)] and nonlateralized PD pathologies [frontal dopamine and perfusion levels (Eidelberg et al., 1995; Kaasinen et al., 2000)]. Third, the hand-switch effect was present when patients 


\begin{tabular}{|c|c|c|c|c|c|c|c|c|}
\hline \multirow[b]{3}{*}{ Cerebral effects } & \multirow[b]{3}{*}{ Anatomical region } & \multirow{2}{*}{\multicolumn{3}{|c|}{ MNI coordinates }} & \multicolumn{4}{|c|}{ Partial correlation } \\
\hline & & & & & \multicolumn{2}{|c|}{ UPDRS-hand } & \multicolumn{2}{|c|}{ UPDRS-foot } \\
\hline & & $x$ & $y$ & $z$ & $r$ & $p$ value & $r$ & $p$ value \\
\hline \multirow[t]{2}{*}{ Switch-related activity } & Cingulate motor area & 4 & -2 & 46 & 0.75 & 0.001 & 0.18 & 0.50 \\
\hline & Left globus pallidus & 18 & -2 & -2 & -0.70 & 0.003 & -0.38 & 0.15 \\
\hline \multirow[t]{2}{*}{ Switch-related connectivity with cingulate motor area } & Left globus pallidus & -20 & 4 & 2 & -0.70 & 0.003 & -0.15 & 0.58 \\
\hline & Left putamen & -22 & 4 & 2 & -0.80 & $<0.001$ & -0.27 & 0.31 \\
\hline
\end{tabular}

Partial correlations between cerebral effects (switch-related activity; connectivity with cingulate motor area) and lateralized disease severity indexes (UPDRS-hand; UPDRS-foot). The four regions reported in this table were significantly correlated with lateralized disease severity (corrected for multiple comparisons, see Results). Post hoc, we calculated partial correlations between the local maxima of these regions and hand-specific disease severity (while correcting for foot-specific disease severity), as well as with foot-specific disease severity (while correcting for hand-specific disease severity). The results show that the brain- behavior relationships are specific for hand-specific disease severity. Note that the $r$ and $p$ values in this table are not corrected for multiple comparisons, so they should be interpreted with caution.

selected actions based on internal context (experiments 1-2), but not when their actions were spatially guided (experiment 3 ).

The hand-switch effect in PD is compatible with computational models suggesting a role of the BG in updating novel representations (O'Reilly, 2006). Impaired updating in PD may be caused by difficulties to select actions that were previously irrelevant (Moustafa et al., 2008), or by impaired inhibition of previously selected actions, allowing them to compete with novel motor representations (Rubchinsky et al., 2003). The latter account is consistent with the reduced switch-related activity observed in the globus pallidus: given the inhibitory effect of the globus pallidus onto the cortex, focused action selection involves the excitation of a large pallidal territory surrounding a functional center, while concurrent inhibition of the functional center allows specific recruitment of cortical representations (Mink, 1996). The net effect should be an increase in pallidal activity, which we only observed in very mildly affected patients. Our findings are also consistent with the observation that further disruption of pallidal function in $\mathrm{PD}$, as caused by pallidotomy, selectively disturbs switching behavior (Tröster et al., 2002).

In contrast to accounts focused on predefined sequences of movements (Benecke et al., 1987; Harrington and Haaland, 1991; Hayes et al., 1998), our results suggest that the BG are implicated during internal selection of independent action plans that depart from prior motor history. This explanatory framework can account for a variety of PD symptoms. For instance, the impairments of PD patients in initiating internally generated movements (Brown and Marsden, 1988) can be seen as a reflection of altered ability to select a change from the previously held posture. Similarly, the slowing and cessation of movements ("freezing") that occurs when patients have to switch from one motor program to another (e.g., when turning during walking) (Snijders et al., 2007) could be related to altered switching abilities.

Contributions of the frontostriatal system to action switching Switching toward the most affected hand evoked brain activity along portions of the medial frontal cortex directly connected with the BG, namely the CMA (Takada et al., 2001) and pre-SMA (Akkal et al., 2007). The CMA response was specific in four different aspects. First, the response was driven by trials requiring switches to the most affected hand, but not to the least affected hand. Second, the CMA response was not sensitive to error processing, which separates this region from neighboring areas involved in performance monitoring (Rushworth et al., 2004; Mars et al., 2005). Third, the CMA response was anatomically confined to a region previously associated with hand movements (Paus, 2001), motor imagery (Ehrsson et al., 2003) and action switching (Rushworth et al., 2002). Fourth, the CMA response was modulated by disease severity, and reciprocally related to the hand- switch effects measured in the BG (i.e., it was particularly strong in those patients with weak or absent BG responses).

The enhanced CMA activity might compensate for reduced BG contributions to action switching. This interpretation is consistent with findings in healthy subjects showing inverse contributions of the striatal and mediofrontal dopamine systems during sequence learning (Garraux et al., 2007). It has been suggested that the striatal and frontal dopamine systems operate on different time scales, with slower and more prolonged neurotransmission in the frontal cortex, probably related to slower dopamine clearance (Lavin et al., 2005). These temporal differences may reflect the nature of the computations that are performed in the striatum (simple associations between actions and expected rewards) and the frontal cortex [exhaustive explorations of possible future outcomes; (Daw et al., 2005)]. Accordingly, these neurochemical and computational properties suggests a functional division of labor between a rapid contextual updating (supported by the BG) and a slower contextual updating, mediated by the frontal cortex (Pasupathy and Miller, 2005; O'Reilly, 2006). In this framework, the increased behavioral hand-switch costs fit with an asymmetrically reduced contribution of the fast striatal system, an increased reliance on the slower mediofrontal system, and a modification of the coupling between these two systems. The reduced corticostriatal coupling may have led to an imbalance between the two systems in the PD patients, biasing the system toward slow updating. This idea is consistent with findings in healthy subjects, in which transient dopamine depletion led to increased behavioral switch costs and diminished functional connectivity between anterior striatum and frontal cortex (Nagano-Saito et al., 2008).

Alternatively, the enhanced CMA activity may represent a pathological response, related to reduced focusing abilities of the BG (Mink, 1996; Rubchinsky et al., 2003). Specifically, reduced switch-related activity in the globus pallidus may have led to impaired inhibitory control over the cortex, as evidenced by the decrease in corticostriatal coupling we observed. In this framework, the enhanced behavioral hand-switch costs may be caused by competition between the previously selected motor representation and the novel one. Further studies, based on interference methods, could distinguish between the two possibilities outlined above.

In contrast to the activity profile observed in the CMA, the pre-SMA responded during both hand-switch trials and error responses, confirming an overlap between performance monitoring (error processing) and action selection in this region (Ullsperger and von Cramon, 2001; Mars et al., 2005). This finding is consistent with several studies suggesting that the pre-SMA monitors and integrates internal states over different trials (Rushworth et al., 2004), switching to alternative action sets when nec- 
essary (Isoda and Hikosaka, 2007; Mars et al., 2007). Accordingly, the enhanced pre-SMA activity we observed could be related to increased cognitive control in the face of reduced focusing abilities of the BG, an instance of the increased contribution of this region to performance monitoring in $\mathrm{PD}$ patients (Catalan et al., 1999; Carbon et al., 2007).

\section{Interpretational issues}

We did not systematically explore how the hand-switch effects vary as a function of the temporal delay between subsequent trials, but it appears that the shorter intertrial intervals in experiment 2 (compared with experiment 1) evoked higher switchcosts that were visible for both hands (while this effect was lateralized in experiment 1). Although these differences might result from a variety of procedural differences between experiments 1 and 2 (see Materials and Methods), it is tempting to speculate that PD patients are particularly impaired when rapid switches between motor plans are required.

Given that in experiments 1-2 subjects responded with their left and right foot, one might argue that the hand-switch effects we report are caused by the execution of foot movements. This appears unlikely for the following reasons. First, the switchrelated activity was confined to a hand-specific portion of the CMA (Paus, 2001). Second, the PD patients were selected for having mainly hand-related symptoms, and accordingly had greater disease severity and motor asymmetry in the hands than in the feet. Given that there were no hand-switch effects when the patients performed spatially cued hand movements (experiment 3 ), it is unlikely that a simple button press with the feet (experiments 1-2) caused the switch effects we report. Third, switchrelated activity in the CMA and in the left BG showed high partial correlations with hand-specific disease severity scores, but not with foot-specific disease severity scores.

Finally, it is possible that $\mathrm{PD}$-related impairments outside the BG may have contributed to the effects we observed (Shin et al., 2005).

\section{Conclusion}

We conclude that striatal dysfunction in PD leads to slower selection of action plans that deviate from the previous motor context, and to correspondingly enhanced medial frontal activity. Our results qualify the general notion that $\mathrm{PD}$ patients are impaired in "internally selecting" actions (Brown and Marsden, 1988), by defining it as a BG-dependent interaction between the recently selected and the current action plan.

\section{References}

Akkal D, Dum RP, Strick PL (2007) Supplementary motor area and presupplementary motor area: targets of basal ganglia and cerebellar output. J Neurosci 27:10659-10673.

Behrens TE, Woolrich MW, Walton ME, Rushworth MF (2007) Learning the value of information in an uncertain world. Nat Neurosci 10:1214-1221.

Benecke R, Rothwell JC, Dick JP, Day BL, Marsden CD (1987) Disturbance of sequential movements in patients with Parkinson's disease. Brain 110:361-379.

Brown RG, Marsden CD (1988) Internal versus external cues and the control of attention in Parkinson's disease. Brain 111:323-345.

Carbon M, Felice Ghilardi M, Dhawan V, Eidelberg D (2007) Correlates of movement initiation and velocity in Parkinson's disease: A longitudinal PET study. Neuroimage 34:361-370.

Catalan MJ, Ishii K, Honda M, Samii A, Hallett M (1999) A PET study of sequential finger movements of varying length in patients with Parkinson's disease. Brain 122:483-495.
Chen PY, Popovich PM (2002) Correlation: parametric and nonparametric measures. Thousand Oaks, CA: Sage.

Cisek P, Kalaska JF (2004) Neural correlates of mental rehearsal in dorsal premotor cortex. Nature 431:993-996.

Cools AR, van den Bercken JH, Horstink MW, van Spaendonck KP, Berger HJ (1984) Cognitive and motor shifting aptitude disorder in Parkinson's disease. J Neurol Neurosurg Psychiatry 47:443-453.

Daw ND, Niv Y, Dayan P (2005) Uncertainty-based competition between prefrontal and dorsolateral striatal systems for behavioral control. Nat Neurosci 8:1704-1711.

de Lange FP, Helmich RC, Toni I (2006) Posture influences motor imagery: an fMRI study. Neuroimage 33:609-617.

de Lange FP, Jensen O, Bauer M, Toni I (2008) Interactions between posterior gamma and frontal alpha/beta oscillations during imagined actions. Front Hum Neurosci 2:7.

Dubois B, Slachevsky A, Litvan I, Pillon B (2000) The FAB: a Frontal Assessment Battery at bedside. Neurology 55:1621-1626.

Ehrsson HH, Geyer S, Naito E (2003) Imagery of voluntary movement of fingers, toes, and tongue activates corresponding body-part-specific motor representations. J Neurophysiol 90:3304-3316.

Eidelberg D, Moeller JR, Ishikawa T, Dhawan V, Spetsieris P, Chaly T, Belakhlef A, Mandel F, Przedborski S, Fahn S (1995) Early differential diagnosis of Parkinson's disease with 18F-fluorodeoxyglucose and positron emission tomography. Neurology 45:1995-2004.

Friston KJ (2007) Statistical parametric mapping: the analysis of functional brain images. Amsterdam: Elsevier/Academic.

Friston KJ, Holmes A, Poline JB, Price CJ, Frith CD (1996) Detecting activations in PET and fMRI: levels of inference and power. Neuroimage 4:223-235.

Garraux G, Peigneux P, Carson RE, Hallett M (2007) Task-related interaction between basal ganglia and cortical dopamine release. J Neurosci 27:14434-14441.

Gentili R, Cahouet V, Ballay Y, Papaxanthis C (2004) Inertial properties of the arm are accurately predicted during motor imagery. Behav Brain Res 155:231-239.

Graybiel AM (1998) The basal ganglia and chunking of action repertoires. Neurobiol Learn Mem 70:119-136.

Harrington DL, Haaland KY (1991) Sequencing in Parkinson's disease. Abnormalities in programming and controlling movement. Brain 114:99-115.

Hayes AE, Davidson MC, Keele SW, Rafal RD (1998) Toward a functional analysis of the basal ganglia. J Cogn Neurosci 10:178-198.

Helmich RC, de Lange FP, Bloem BR, Toni I (2007) Cerebral compensation during motor imagery in Parkinson's disease. Neuropsychologia 45:2201-2215.

Isoda M, Hikosaka O (2007) Switching from automatic to controlled action by monkey medial frontal cortex. Nat Neurosci 10:240-248.

Jahanshahi M, Jenkins IH, Brown RG, Marsden CD, Passingham RE, Brooks DJ (1995) Self-initiated versus externally triggered movements. I. An investigation using measurement of regional cerebral blood flow with PET and movement-related potentials in normal and Parkinson's disease subjects. Brain 118:913-933.

Jahanshahi M, Profice P, Brown RG, Ridding MC, Dirnberger G, Rothwell JC (1998) The effects of transcranial magnetic stimulation over the dorsolateral prefrontal cortex on suppression of habitual counting during random number generation. Brain 121:1533-1544.

Jeannerod M (2006) Motor cognition: what actions tell to the self. Oxford: Oxford UP.

Jueptner M, Frith CD, Brooks DJ, Frackowiak RS, Passingham RE (1997) Anatomy of motor learning. II. Subcortical structures and learning by trial and error. J Neurophysiol 77:1325-1337.

Kaasinen V, Någren K, Hietala J, Oikonen V, Vilkman H, Farde L, Halldin C, Rinne JO (2000) Extrastriatal dopamine D2 and D3 receptors in early and advanced Parkinson's disease. Neurology 54:1482-1487.

Lalo E, Thobois S, Sharott A, Polo G, Mertens P, Pogosyan A, Brown P (2008) Patterns of bidirectional communication between cortex and basal ganglia during movement in patients with Parkinson disease. J Neurosci 28:3008-3016.

Langston JW, Widner H, Goetz CG, Brooks D, Fahn S, Freeman T, Watts R (1992) Core assessment program for intracerebral transplantations (CAPIT). Mov Disord 7:2-13.

Lavin A, Nogueira L, Lapish CC, Wightman RM, Phillips PE, Seamans JK 
(2005) Mesocortical dopamine neurons operate in distinct temporal domains using multimodal signaling. J Neurosci 25:5013-5023.

Lund TE, Nørgaard MD, Rostrup E, Rowe JB, Paulson OB (2005) Motion or activity: their role in intra- and inter-subject variation in fMRI. Neuroimage 26:960-964.

Mars RB, Coles MG, Grol MJ, Holroyd CB, Nieuwenhuis S, Hulstijn W, Toni I (2005) Neural dynamics of error processing in medial frontal cortex. Neuroimage 28:1007-1013.

Mars RB, Piekema C, Coles MG, Hulstijn W, Toni I (2007) On the programming and reprogramming of actions. Cereb Cortex 17:2972-2979.

Mattay VS, Tessitore A, Callicott JH, Bertolino A, Goldberg TE, Chase TN, Hyde TM, Weinberger DR (2002) Dopaminergic modulation of cortical function in patients with Parkinson's disease. Ann Neurol 51:156-164.

McFarland NR, Haber SN (2000) Convergent inputs from thalamic motor nuclei and frontal cortical areas to the dorsal striatum in the primate. J Neurosci 20:3798-3813.

Mink JW (1996) The basal ganglia: focused selection and inhibition of competing motor programs. Prog Neurobiol 50:381-425.

Moustafa AA, Sherman SJ, Frank MJ (2008) A dopaminergic basis for working memory, learning and attentional shifting in Parkinsonism. Neuropsychologia 46:3144-3156.

Mueller VA, Brass M, Waszak F, Prinz W (2007) The role of the preSMA and the rostral cingulate zone in internally selected actions. Neuroimage 37:1354-1361.

Nagano-Saito A, Leyton M, Monchi O, Goldberg YK, He Y, Dagher A (2008) Dopamine depletion impairs frontostriatal functional connectivity during a set-shifting task. J Neurosci 28:3697-3706.

Nichols T, Brett M, Andersson J, Wager T, Poline JB (2005) Valid conjunction inference with the minimum statistic. Neuroimage 25:653-660.

O'Reilly RC (2006) Biologically based computational models of high-level cognition. Science 314:91-94.

Parsons LM (1987) Imagined spatial transformations of one's hands and feet. Cogn Psychol 19:178-241.

Pasupathy A, Miller EK (2005) Different time courses of learning-related activity in the prefrontal cortex and striatum. Nature 433:873-876.

Paus T (2001) Primate anterior cingulate cortex: where motor control, drive and cognition interface. Nat Rev Neurosci 2:417-424.

Picard N, Strick PL (1996) Motor areas of the medial wall: a review of their location and functional activation. Cereb Cortex 6:342-353.

Polit A, Bizzi E (1979) Characteristics of motor programs underlying arm movements in monkeys. J Neurophysiol 42:183-194.
Redgrave P, Gurney K (2006) The short-latency dopamine signal: a role in discovering novel actions? Nat Rev Neurosci 7:967-975.

Ridderinkhof KR, Ullsperger M, Crone EA, Nieuwenhuis S (2004) The role of the medial frontal cortex in cognitive control. Science 306:443-447.

Rosenbaum DA, Meulenbroek RJ, Vaughan J, Jansen C (2001) Posturebased motion planning: applications to grasping. Psychol Rev 108:709-734.

Rosenbaum DA, Cohen RG, Jax SA, Weiss DJ, van der Wel R (2007) The problem of serial order in behavior: Lashley's legacy. Hum Mov Sci 26:525-554.

Rubchinsky LL, Kopell N, Sigvardt KA (2003) Modeling facilitation and inhibition of competing motor programs in basal ganglia subthalamic nucleus-pallidal circuits. Proc Natl Acad Sci U S A 100:14427-14432.

Rushworth MF, Hadland KA, Paus T, Sipila PK (2002) Role of the human medial frontal cortex in task switching: a combined fMRI and TMS study. J Neurophysiol 87:2577-2592.

Rushworth MF, Walton ME, Kennerley SW, Bannerman DM (2004) Action sets and decisions in the medial frontal cortex. Trends Cogn Sci $8: 410-417$.

Shenton JT, Schwoebel J, Coslett HB (2004) Mental motor imagery and the body schema: evidence for proprioceptive dominance. Neurosci Lett 370:19-24.

Shin JC, Aparicio P, Ivry RB (2005) Multidimensional sequence learning in patients with focal basal ganglia lesions. Brain Cogn 58:75-83.

Snijders AH, van de Warrenburg BP, Giladi N, Bloem BR (2007) Neurological gait disorders in elderly people: clinical approach and classification. Lancet Neurol 6:63-74.

Takada M, Tokuno H, Hamada I, Inase M, Ito Y, Imanishi M, Hasegawa N, Akazawa T, Hatanaka N, Nambu A (2001) Organization of inputs from cingulate motor areas to basal ganglia in macaque monkey. Eur J Neurosci 14:1633-1650.

Tröster AI, Woods SP, Fields JA, Hanisch C, Beatty WW (2002) Declines in switching underlie verbal fluency changes after unilateral pallidal surgery in Parkinson's disease. Brain Cogn 50:207-217.

Tzourio-Mazoyer N, Landeau B, Papathanassiou D, Crivello F, Etard O, Delcroix N, Mazoyer B, Joliot M (2002) Automated anatomical labeling of activations in SPM using a macroscopic anatomical parcellation of the MNI MRI single-subject brain. Neuroimage 15:273-289.

Ullsperger M, von Cramon DY (2001) Subprocesses of performance monitoring: a dissociation of error processing and response competition revealed by event-related fMRI and ERPs. Neuroimage 14:1387-1401. 